\title{
Antenna Array Design in MIMO Radar Using NSK Polynomial Factorization Algorithm
}

\author{
Shuainan Gu, Ke Li, Xiukun Ren, and Na-e Zheng \\ China National Digital Switching System Engineering and Technological Research Center, Zhengzhou 450002, China \\ Correspondence should be addressed to Shuainan Gu; m15690887855@163.com
}

Received 15 September 2015; Revised 9 December 2015; Accepted 17 December 2015

Academic Editor: Ahmed A. Kishk

Copyright (C) 2016 Shuainan Gu et al. This is an open access article distributed under the Creative Commons Attribution License, which permits unrestricted use, distribution, and reproduction in any medium, provided the original work is properly cited.

\begin{abstract}
The work presented here is concerned with the antenna array design in collocated multiple-input multiple-output (MIMO) radars. After knowing the system requirements, the antenna array design problem is formulated as a standard polynomial factorization. In addition, an algorithm based on Newton-Schubert-Kronecker (NSK) polynomial factorization is proposed. The algorithm contains three steps. First, linear factors are extracted by extended Vieta theorem. Then, undermined high-order factors are confirmed with Newton interpolation and certain high-order factors should be searched for within the undermined ones. Finally, the antenna array configurations are determined according to the result of polynomial factorization. Simulations confirm the wide use of the proposed algorithm in MIMO radar antenna array design.
\end{abstract}

\section{Introduction}

Radar theory has developed quickly for the last fifty years and multiple-input multiple-output (MIMO) radar has been introduced as a new concept in the design of radar systems [1-5], which has received considerable attention, for it performs better than conventional radars. MIMO radar transmits multiple signals via its antennas. At each receiving channel, every target response can be selected with a matched filter [6-8]. In this way, an extended target space-sample can be reached by the array design of the transmitting and receiving aperture, which provides a potential for the system to have a higher spatial sample ability and space resolution than a conventional phase array system [9]. The array configurations influence the system performance directly.

In the paper, we consider the antenna array design of collocated MIMO radar. For convenient and simple description, the kind of collocated MIMO radar whose transmitting and receiving antenna arrays share no antennas is called bistatic MIMO radar, and the other kind of collocated MIMO radar whose transmitting and receiving antenna arrays share the same antenna array is called monostatic MIMO radar.

Compared with the traditional array design methods, MIMO radar arrangement needs to synthesize transmitting and receiving arrays [10-12]. In [13, 14], a possible simplified algorithm is presented in order to find MIMO array setups of maximum dimension with minimum redundancy. In [15], two different MIMO radar array designs are described, which aims at obtaining optimal angular resolution and controlling the side lobes of the synthetic array pattern. A hybrid method based on cyclic difference sets and genetic algorithm is proposed in [16] to design antenna arrays in order to synthesize the MIMO minimum variance distortionless response (MVDR). The method can provide a large set of array design solutions at small computational cost which may greatly enrich the choice of the proper array configurations in antenna engineering applications. MIMO radar array design is analyzed based on the spatial convolution principle in [9]. Whether a given array is a virtual equivalent MIMO radar array or not is dependent on whether the given array can be separated into two arrays with the spatial convolution. However, the algorithm is ineffective in bistatic MIMO radar. Then the polynomial factorization method is proposed in [17-20] to obtain the desired virtual equivalent MIMO radar antenna array. There may be multiple solutions for the transmitting and receiving antenna arrays and different solutions may have different array performance. Adaptive beamforming algorithm is used to comparatively analyze the statistical 

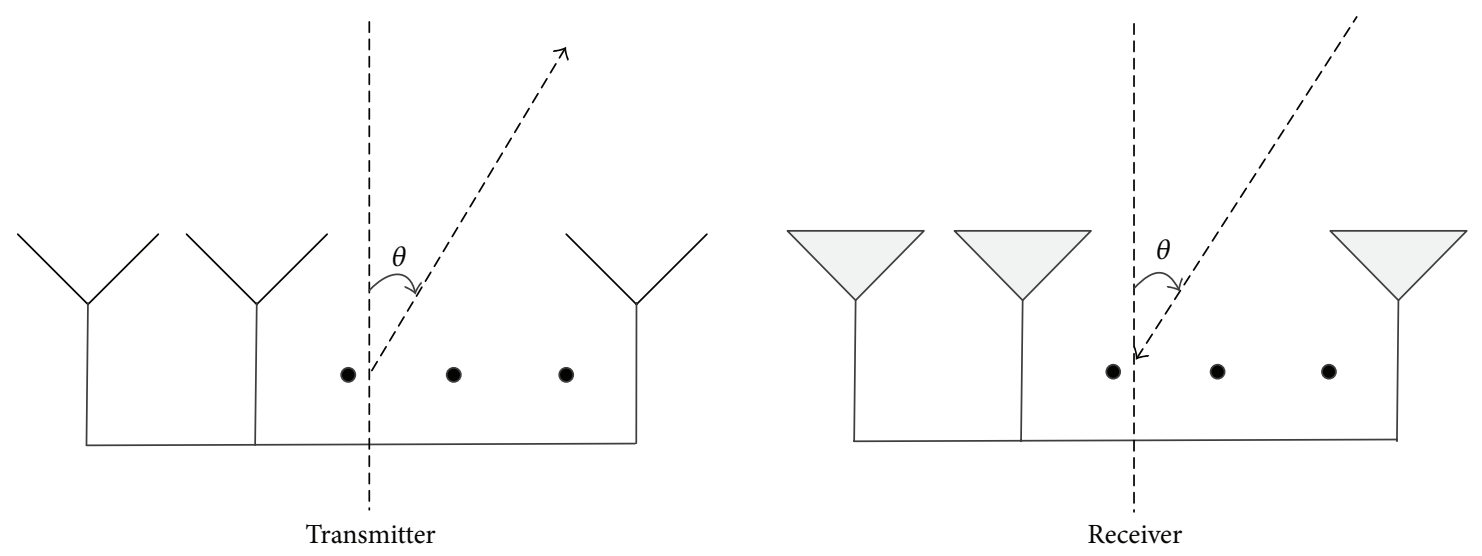

FIGURE 1: Signal model of collocated MIMO radar.

output signal-to-interference-plus-noise ratio (SINR) performance of the designed arrays in [17]. However, it is applicable only when the virtual MIMO antenna array is uniform. Nonuniform virtual MIMO antenna array design needs to be investigated in the subsequent work.

Following the developments of $[9,17]$, a MIMO radar array design algorithm using NSK polynomial factorization is proposed which aims to find all the possible decompositions of a system virtual equivalent MIMO radar array into transmitting and receiving arrays. Simulation results indicate that the proposed algorithm can be widely used no matter whether the transmitting and receiving arrays are bistatic or collocated and the system equivalent antenna array is nonuniform or uniform.

The rest of the paper is organized as follows: Section 2 develops a signal model of MIMO radar. Section 3 proposes the array design algorithm using NSK polynomial factorization. Section 4 analyzes the design examples of different types of MIMO radar and examines the simulation performance. Section 5 provides the summary and concluding remarks.

\section{System Model}

The narrow-band signal and far-field scenarios are considered in this paper, assuming the transmitting and receiving arrays of MIMO radar are collocated and are both linear arrays. Consider a MIMO radar system with a transmitter equipped with $M$ collocated antennas which are located at $u_{1}, u_{2}, \ldots, u_{M}$ and a receiver with antennas located at $v_{1}, v_{2}, \ldots, v_{N}$.
Assume there is a far-field point target and let $\theta$ denote the location parameter of the target (see Figure 1). The steering vector for the receiving arrays is denoted by

$$
\begin{aligned}
& \mathbf{A}(\theta)=\left[\exp \left(j \frac{2 \pi v_{1} \sin \theta}{\lambda}\right), \exp \left(j \frac{2 \pi v_{2} \sin \theta}{\lambda}\right), \ldots,\right. \\
& \left.\quad \exp \left(j \frac{2 \pi v_{N} \sin \theta}{\lambda}\right)\right]^{T} .
\end{aligned}
$$
by

The steering vector for the transmitting arrays is denoted

$$
\begin{gathered}
\mathbf{B}(\theta)=\left[\exp \left(j \frac{2 \pi u_{1} \sin \theta}{\lambda}\right), \exp \left(j \frac{2 \pi u_{2} \sin \theta}{\lambda}\right), \ldots,\right. \\
\left.\quad \exp \left(j \frac{2 \pi u_{M} \sin \theta}{\lambda}\right)\right]^{T}
\end{gathered}
$$

According to the formation principle of virtual array, the steering vector by matching the $p$ th receiving antenna and $i$ th transmitting antenna is

$$
c_{p i}(\theta)=\exp \left(j \frac{2 \pi\left(u_{i}+v_{p}\right) \sin \theta}{\lambda}\right) .
$$

The normalized position of the virtual array element is $u_{i}+v_{p}$, and the steering vector for the virtual array can be expressed as

$$
\mathbf{C}(\theta)=\mathbf{A}(\theta) \mathbf{B}^{T}(\theta)=\left[\begin{array}{cccc}
e^{\left(j\left(2 \pi\left(u_{1}+v_{1}\right) \sin \theta / \lambda\right)\right)} & e^{\left(j\left(2 \pi\left(u_{1}+v_{2}\right) \sin \theta / \lambda\right)\right)} & \cdots & e^{\left(j\left(2 \pi\left(u_{1}+v_{N}\right) \sin \theta / \lambda\right)\right)} \\
e^{\left(j\left(2 \pi\left(u_{2}+v_{1}\right) \sin \theta / \lambda\right)\right)} & e^{\left(j\left(2 \pi\left(u_{2}+v_{2}\right) \sin \theta / \lambda\right)\right)} & \cdots & e^{\left(j\left(2 \pi\left(u_{2}+v_{N}\right) \sin \theta / \lambda\right)\right)} \\
\vdots & \vdots & \cdots & \vdots \\
e^{\left(j\left(2 \pi\left(u_{M}+v_{1}\right) \sin \theta / \lambda\right)\right)} & e^{\left(j\left(2 \pi\left(u_{M}+v_{2}\right) \sin \theta / \lambda\right)\right)} & \cdots & e^{\left(j\left(2 \pi\left(u_{M}+v_{N}\right) \sin \theta / \lambda\right)\right)}
\end{array}\right] .
$$


The transmitter transmits orthogonal waveforms, ensuring independent signal channels and avoiding power synthesis of transmitting channels. Orthogonal signals can be separated by matched filters at the receiver. A virtual receiving array with antenna elements located at $\left\{u_{m}+v_{n}\right\}, m=$ $1,2, \ldots, M, n=1,2, \ldots, N$, can be synthesized. If there is an overlapping virtual element at a certain position, the number of the overlapping elements is the equivalent value at the position.

Assuming that the array elements are isotropic, the beam pattern is determined by the array structure and the weighting values [21, 22]. In MIMO radar system, the weighting values are usually all defined as 1 . In that case, the system equivalent radiation beam pattern is decomposed into the product of the transmitter radiation beam pattern and the receiver radiation beam pattern according to [17], so the far-field equivalent antenna radiation beam pattern is expressed as

$$
\begin{aligned}
P_{V}(\theta) & =P_{T}(\theta) P_{R}(\theta) \\
& =\sum_{m=1}^{M} \sum_{n=1}^{N} g_{T}(m) g_{R}(n) e^{j 2 \pi\left(v_{n}+u_{m}\right) \sin \theta / \lambda},
\end{aligned}
$$

where $P_{T}(\theta)=\sum_{m=1}^{M} g_{T}(m) e^{j 2 \pi u_{m} \sin \theta / \lambda}$ and $P_{R}(\theta)=$ $\sum_{n=1}^{N} g_{R}(n) e^{j 2 \pi v_{n} \sin \theta / \lambda}$ denote, respectively, the far-field radiation pattern for the transmitter and receiver. $g_{T}(m)$ and $g_{R}(n)$, respectively, denote the equivalent value at a certain position of transmitting and receiving arrays.

By substituting $x=e^{j 2 \pi d \sin \theta / \lambda}$ with $d$ being the interelement spacing, the equivalent, transmitter, and receiver radiation patterns can be expressed, respectively, as

$$
\begin{aligned}
& P_{T}(x)=\sum_{m=0}^{M-1} g_{T}(m) x^{m}, \\
& P_{R}(x)=\sum_{n=0}^{N-1} g_{R}(n) x^{n}, \\
& P_{V}(x)=\sum_{i=0}^{M+N-2} g_{V}(i) x^{i},
\end{aligned}
$$

where $g_{V}(i)$ denotes equivalent value at the $i$ th position of virtual equivalent array. In MIMO radar systems, the equivalent values have practical significance and they represent the number of antennas at certain positions.

Thus, the equivalent array, transmitting array, and receiving array meet the following relationship:

$$
P_{V}(x)=P_{T}(x) P_{R}(x) .
$$

The polynomial corresponding to the equivalent array is decomposed into the product of two factors which, respectively, correspond to the transmitting and receiving array.

\section{Antenna Array Design in MIMO Radar Using NSK Polynomial Factorization Algorithm}

The MIMO radar antenna array design method can be divided into two steps: the structure of the equivalent array is determined by the beam pattern specification, such as the desired main lobe width and the desired side lobe level. And then the transmitting and receiving arrays can be calculated through NSK polynomial factorization. The first step is the same as the general antenna array design which is not discussed in our paper [23-25]. Our paper focuses on the second step: how to calculate transmitting and receiving arrays by equivalent antenna array.

3.1. Algorithm Design. According to the system requirements, the equivalent array is modeled as a polynomial. Combining Newton's, Schubert's, and Kronecker's core ideas of polynomial factorization, an algorithm is proposed which has wider application than the existing array design methods and we call it Newton-Schubert-Kronecker (NSK) polynomial factorization [26].

In this paper, the standardized formation of the polynomial is defined as

$$
P_{V}(x)=a_{0}^{(0)}+a_{1}^{(0)} x+a_{2}^{(0)} x^{2}+\cdots+a_{n-1}^{(0)} x^{n-1}+x^{n} .
$$

The coefficients $a_{i}^{(0)}$ are integers and the highest coefficient $a_{n}^{(0)}$ equals 1 . If the polynomial is a nonstandardized form, it should be standardized at first.

3.1.1. The Standardization of Polynomial. The standardized formation of the array equivalent vector needs to satisfy the following two requirements when designing MIMO radar array using NSK polynomial factorization: (1) the elements of $\mathbf{G}_{V}$ are positive integers; (2) the highest coefficient $a_{n}^{(0)}$ equals 1 .

If $\mathbf{G}_{V}$ satisfies requirement (1) but does not satisfy requirement (2), the polynomial factorization can be converted to the factorization of a standardized polynomial according to [26].

In fact, elements of the equivalent vector determined by the system performance requirements are not all integers; that is, it does not satisfy requirement (1). In the case, each element of $\mathbf{G}_{V}$ is expanded with the same multiples to be an integer. The following simulation results show that the beam pattern of the decimal equivalent vector is the same as the corresponding integer equivalent vector's.

When $\mathbf{G}_{V}=(0.5,1,2,1.5,1.5,1,0.5)$, there are decimal equivalent coefficients in $\mathbf{G}_{V}$. Expand each element in $\mathbf{G}_{V}(i)$ with four multiples and get $\mathbf{G}_{V}^{\prime}=(1,2,4,3,3,2,1)$. Figure 2 gives the corresponding beam patterns of $\mathbf{G}_{V}$ and $\mathbf{G}_{V}^{\prime}$. The two patterns are the same where the main lobe width is 18.65 degrees and the first side lobe level is $-20.95 \mathrm{~dB}$.

In conclusion, the beam pattern of the decimal equivalent vector is the same as the one of the corresponding integer equivalent vector. When there are decimal equivalent coefficients, expand the elements of the vector with the same multiples so that the elements can become integers firstly. Then, if the highest coefficient $a_{n}^{(0)} \neq 1$, convert the polynomial factorization to a standardized polynomial factorization equivalently according to [26]. Finally, design the antenna array using NSK polynomial factorization algorithm. 


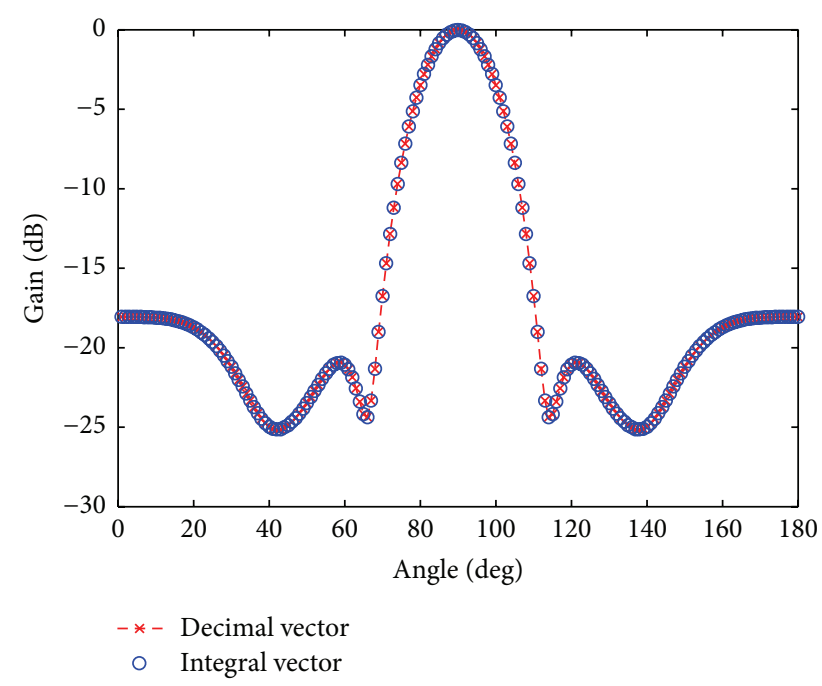

FIgURE 2: Performance of decimal vector and integral vector.

3.1.2. Factors Extraction of Different Powers. Suppose the standardized formation of the polynomial for system equivalent array is

$$
P_{V}(x)=a_{0}^{(0)}+a_{1}^{(0)} x+a_{2}^{(0)} x^{2}+\cdots+a_{n-1}^{(0)} x^{n-1}+x^{n}
$$

The essence of polynomial factorization is to find all of the factors. Thus, from briefness to complexity, the polynomial extraction procedure is given as follows.

(1) The Extraction of $x$. If $a_{0}^{(0)}=a_{1}^{(0)}=\cdots=a_{m-1}^{(0)}=0$ and $a_{m}^{(0)} \neq 0$, it is easy to extract $x^{m}$,

$$
\begin{aligned}
P_{V}^{(1)}(x) & =\frac{P_{V}(x)}{x^{m}} \\
& =a_{0}^{(1)}+a_{1}^{(1)} x+\cdots+a_{n-m-1}^{(1)} x^{n-m-1}+x^{n-m},
\end{aligned}
$$

where $a_{i}^{(1)}=a_{i+m}^{(0)}(i=0,1, \ldots, n-m-1)$.

If $x$ cannot be extracted, then

$$
P_{V}^{(1)}(x)=P_{V}(x)=a_{0}^{(1)}+a_{1}^{(1)} x+\cdots+a_{n-1}^{(1)} x^{n-1}+x^{n},
$$

where $a_{i}^{(1)}=a_{i}^{(0)}(i=0,1, \ldots, n-1)$.

(2) The Extraction of the Linear Factor $x+c_{i}$. Supposing the expression of $P_{V}^{(1)}(x)$ is as (10), the extraction process of $x+c_{i}$ is given as follows (when the expression of $P_{V}^{(1)}(x)$ is as (11), the process is similar).

If the roots of $P_{V}^{(1)}(x)$ are $d_{0}, d_{1}, \ldots, d_{q}$, we can get $\prod_{i=0}^{q} d_{i}=(-1)^{n-m} a_{0}^{(1)}$ through the extended Vieta theorem. In MIMO radar array design, we can try to find the positive factors $c_{0}, c_{1}, \ldots, c_{p}$ of the constant $a_{0}^{(1)}$ and calculate $P_{V}^{(1)}\left(-c_{i}\right)$, $i=0,1,2, \ldots, p$. If $P_{V}^{(1)}\left(-c_{i}\right)=0, i=0,1,2, \ldots, p, x+c_{i}$ is the factor of $P_{V}^{(1)}(x)$ and $P_{V}^{(1)}(x)$ can be expressed as

$$
\begin{aligned}
P_{V}^{(1)}(x) & =x^{n-m}+a_{n-m-1}^{(1)} x^{n-m-1}+\cdots+a_{1}^{(1)} x+a_{0}^{(1)} \\
& =\sum_{k=0}^{n-m-1} a_{k}^{(2)} x^{k}\left(x+c_{i}\right),
\end{aligned}
$$

where

$$
\begin{aligned}
& a_{n-m-1}^{(2)}=1, \\
& a_{n-m-k}^{(2)}=a_{n-m-k+1}^{(1)}-a_{n-m-k+1}^{(2)} \cdot c_{i} \\
& \quad(k=2, \ldots, n-m) .
\end{aligned}
$$

Therefore,

$$
P_{V}^{(2)}(x)=\sum_{k=0}^{n-m-1} a_{k}^{(2)} x^{k}
$$

Try to extract $x+c_{i}$ from $P_{V}^{(2)}(x)$ again until all the linear factors are removed.

Suppose the polynomial is $Q_{V}^{(1)}(x)$ after extraction of $x+c_{i}$ and then extract high-order factors from $Q_{V}^{(1)}(x)$.

(3) High-Order Factors Extraction. If $\operatorname{deg}\left(Q_{V}^{(1)}(x)\right)=N_{\max }$ (deg denotes the degree of $Q_{V}^{(1)}(x)$ ) and $Q_{V}^{(1)}(x)$ can be factorized, there is at least one factor whose degree is not more than $N_{\max } / 2$. Define $s=\left\lfloor N_{\max } / 2\right\rfloor$ and the degree $k$ of the factor $g_{k}^{\prime}(x)$ meets the following relationship: $k=$ $\operatorname{deg}\left(g_{k}^{\prime}(x)\right) \leq s$. The undermined factors $g_{k}(x)$ can be obtained by Newton interpolation.

The concrete process is given as follows:

(a) Supposing $k+1$ different integers $x_{0}, x_{1}, \ldots, x_{k}$, calculate $Q_{V}^{(k-1)}\left(x_{0}\right), Q_{V}^{(k-1)}\left(x_{1}\right), \ldots, Q_{V}^{(k-1)}\left(x_{k}\right)$.

(b) If $g_{k}(x)$ is the factor of $Q_{V}^{(k-1)}(x), g_{k}\left(x_{i}\right)$ must satisfy $g_{k}\left(x_{i}\right) \mid Q_{V}^{(k-1)}\left(x_{i}\right)$. For $Q_{V}^{(k-1)}\left(x_{i}\right)$ has limited factors, the number of the vectors $\left[g_{k}(0), g_{k}(1), \ldots, g_{k}(k)\right]$ with dimension $k+1$ is also limited.

(c) For each vector $\left[g_{k}(0), g_{k}(1), \ldots, g_{k}(k)\right]$, a $k$-order polynomial $g_{k}(x)$ can be only determined by interpolation formula. When $k$ increases from 2 to $s$, the interpolating point increases. In order to utilize the results calculated, Newton interpolation formula is the best choice:

$g_{k}(x)$

$$
\begin{aligned}
= & g(0)+g(0,1)(x-0)+\cdots \\
& +g(0,1, \ldots, k)(x-0)(x-1) \cdots(x-(k-1)),
\end{aligned}
$$

where

$$
\begin{aligned}
& g(0,1, \ldots, m) \\
& =\sum_{j=0}^{m} \frac{g_{k}\left(x_{j}\right)}{\left(x_{j}-x_{0}\right) \cdots\left(x_{j}-x_{j-1}\right)\left(x_{j}-x_{j+1}\right) \cdots\left(x_{j}-x_{m}\right)} .
\end{aligned}
$$


The undermined factors $g_{k}(x)$ can be obtained and $g_{k}^{\prime}(x)$ exists among them. Then, update $Q_{V}^{(k-1)}(x)$ to get $Q_{V}^{(k)}(x)$ by removing all $g_{k}^{\prime}(x)$, which satisfies $Q_{V}^{(k-1)}(x)=Q_{V}^{(k)}(x) g_{k}^{\prime}(x)$. So when $k$ increases from 2 to $s$ sequentially, all the factors $g_{k}^{\prime}(x)$ of $Q_{V}^{(1)}(x)$ can be obtained.

By the results of polynomial factorization, the polynomial of the system equivalent array can be expressed as the product of two factors. The MIMO radar antenna array is designed according to the two factors.

In total, the process of the proposed algorithm is shown as follows.

Process of Antenna Array Design in MIMO Radar Using NSK Polynomial Factorization Algorithm

Step 1 (initialization). Determine the standardized polynomial $P_{V}(x)$ by the requirements of system performance.

Step 2 (judgment on requirements of $x$ extraction). If the requirements are satisfied, extract $x$; otherwise go to Step 3 .

Step 3 (judgment on requirements of $x+c_{i}$ ). If the requirements are satisfied, extract $x+c_{i}$ and go to Step 3 again; otherwise go to Step 4.

Step 4. Judge the degree of $Q_{V}^{(1)}(x)$ and calculate $s=$ $\left\lfloor N_{\max } / 2\right\rfloor$. If $s \geq 4$, go to Step 5; otherwise end the algorithm.

Step 5 (extraction of high-order factors). $K$-order undermined factors $g_{k}(x), k \in\{2,3, \ldots, s\}$, are determined by Newton interpolation. Search among $g_{k}(x)$ and determine the factors $g_{k}^{\prime}(x)$ of $Q_{V}^{(k-1)}(x)$. Update $Q_{V}^{(k-1)}(x)$ to get $Q_{V}^{(k)}(x)$ by removing all $g_{k}^{\prime}(x)$. When $k>s$, the algorithm ends.

Step 6. Determine the array configurations according to the results of polynomial factorization.

3.2. Design Example. In order to facilitate the understanding of MIMO radar array design algorithm based on NSK polynomial factorization, the following example is given.

Assuming that the equivalent value determined by the requirements of system performance is $\mathbf{G}_{V}=(1,0.5,2$, $2,1.5,1.5,0.5)$, then the polynomial is $1+0.5 x+2 x^{2}+2 x^{3}+$ $1.5 x^{4}+1.5 x^{5}+0.5 x^{6}$.

The corresponding integer equivalent value is $\mathbf{G}_{V}^{\prime}=(2,1$, $4,4,3,3,1)$ and the polynomial is $2+x+4 x^{2}+4 x^{3}+3 x^{4}+$ $3 x^{5}+x^{6}$ by standardization.

(1) The Extraction of $x$. For $a_{0}^{(0)}=2 \neq 0, x$ cannot be extracted from the polynomial; then $P_{V}^{(1)}(x)=P_{V}(x)$.

(2) The Extraction of $x+c_{i}$. The constant $a_{0}^{(0)}$ contains the positive factors 1 and 2. For $P_{V}^{(1)}(-1) \neq 0$ and $P_{V}^{(1)}(-2)=0, x+$ 2 is the factor of $P_{V}^{(1)}(x)$. Consider $P_{V}^{(1)}(x)=(2+x)\left(1+2 x^{2}+\right.$ $\left.x^{3}+x^{4}+x^{5}\right)$; then $P_{V}^{(2)}(x)=1+2 x^{2}+x^{3}+x^{4}+x^{5}$.

Try to extract $x+c_{i}$ from $P_{V}^{(2)}(x)$ again. The constant of $P_{V}^{(2)}(x)$ is 1 and the constant contains the positive factor 1 . For
$P_{V}^{(2)}(-1) \neq 0, P_{V}^{(2)}(x)$ cannot be extracted by $x+c_{i}$. Consider $Q_{V}^{(1)}(x)=P_{V}^{(2)}(x)=1+2 x^{2}+x^{3}+x^{4}+x^{5}$.

(3) High-Order Factors Extraction. Given $Q_{V}^{(1)}(x)=1+2 x^{2}+$ $x^{3}+x^{4}+x^{5}$, we can know $N_{\max }=\operatorname{deg}\left(Q_{V}^{(1)}(x)\right)=5$, and $s=\left\lfloor N_{\max } / 2\right\rfloor=2$. Then the undetermined quadratic polynomials can be obtained by the Newton interpolating method as follows:

(a) Calculate $Q_{V}^{(1)}(0)=1, Q_{V}^{(1)}(1)=6$, and $Q_{V}^{(1)}(2)=65$.

(b) Possible values of $g_{2}(0), g_{2}(1)$, and $g_{2}(2)$ are $1,-1 ; 1$, $-1,2,-2,3,-3,6$, and -6 ; and $1,-1,5,-5,13,-13,65$, and -65 , respectively. There are 128 combinations in total about the values of $g_{2}(0), g_{2}(1)$, and $g_{2}(2)$, which are $(1,1,1),(1,1,-1),(1,1,5), \ldots,(-1,-6,-65)$.

(c) The corresponding undetermined quadratic polynomials for each combination are calculated by Newton interpolation and then search among all the undetermined ones to obtain the determined quadratic polynomials of $Q_{V}^{(1)}(x)$. The interpolation polynomial corresponding to the combination of $(1,2,5)$ is $g_{2}(x)=x^{2}+1$, by which $Q_{V}^{(1)}(x)$ can be divided, so $g_{2}^{\prime}(x)=g(x)$.

We can get the final results of the polynomial factorization: $P_{V}(x)=(x+2)\left(x^{2}+1\right)\left(x^{3}+x^{2}+1\right)$.

According to the results above, 6 kinds of MIMO radar array designs can be obtained as follows, and system equivalent arrays of these 6 designs are nonuniform:

$$
\begin{aligned}
& \text { Design 1: } P_{T}(x)=2+x, P_{R}(x)=\left(x^{2}+1\right)\left(x^{3}+x^{2}+1\right) . \\
& \text { Design 2: } P_{T}(x)=1+x^{2}, P_{R}(x)=(x+1)\left(x^{3}+x^{2}+1\right) . \\
& \text { Design 3: } P_{T}(x)=x^{3}+x^{2}+1, P_{R}(x)=(x+2)\left(x^{2}+1\right) . \\
& \text { Design 4: } P_{T}(x)=\left(x^{2}+1\right)\left(x^{3}+x^{2}+1\right), P_{R}(x)=2+x . \\
& \text { Design 5: } P_{T}(x)=(x+2)\left(x^{3}+x^{2}+1\right), P_{R}(x)=1+x^{2} . \\
& \text { Design 6: } P_{T}(x)=(x+2)\left(x^{2}+1\right), P_{R}(x)=x^{3}+x^{2}+1 .
\end{aligned}
$$

\section{Design Examples and Performance Simulation}

In this section, the performance of the proposed algorithm is simulated and analyzed. Without loss of generality, assume that all array antennas are omnidirectional and the target is located in the far-field. In order to compare the application scopes of different algorithms, the antenna array design for different types of MIMO radar is discussed. In the MIMO radar system, the uniform equivalent system arrays cannot be obtained by monostatic transmitting and receiving arrays. So the following three types of MIMO radar are considered.

Example 1 (nonuniform equivalent array design of monostatic MIMO radar). Consider $\mathbf{G}_{V}=(1,2,3,2,1)$ and $P_{V}(x)=1+2 x+3 x^{2}+2 x^{3}+x^{4}$.

The equivalent array is nonuniform, so the algorithm in [17] cannot be applied. 

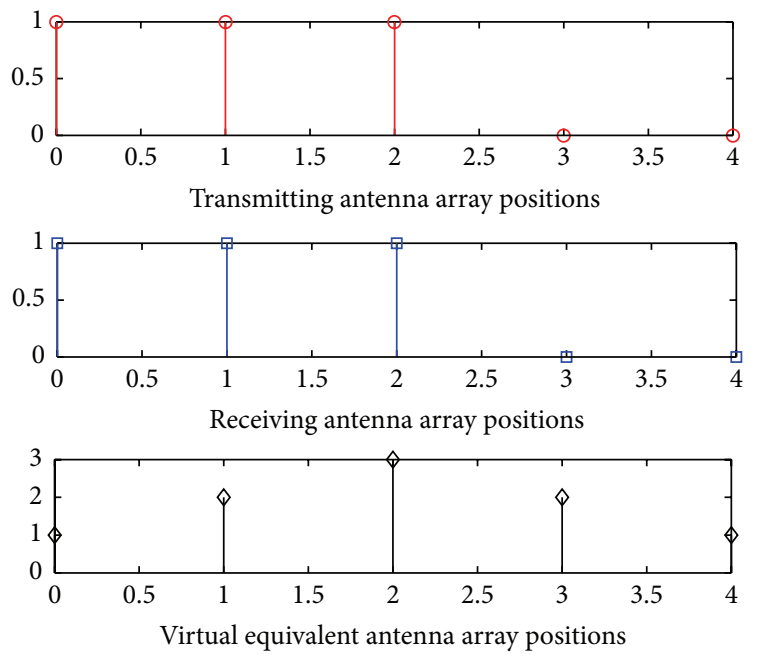

FIGURE 3: Antenna array designs of NSK polynomial factorization and inverse convolution.

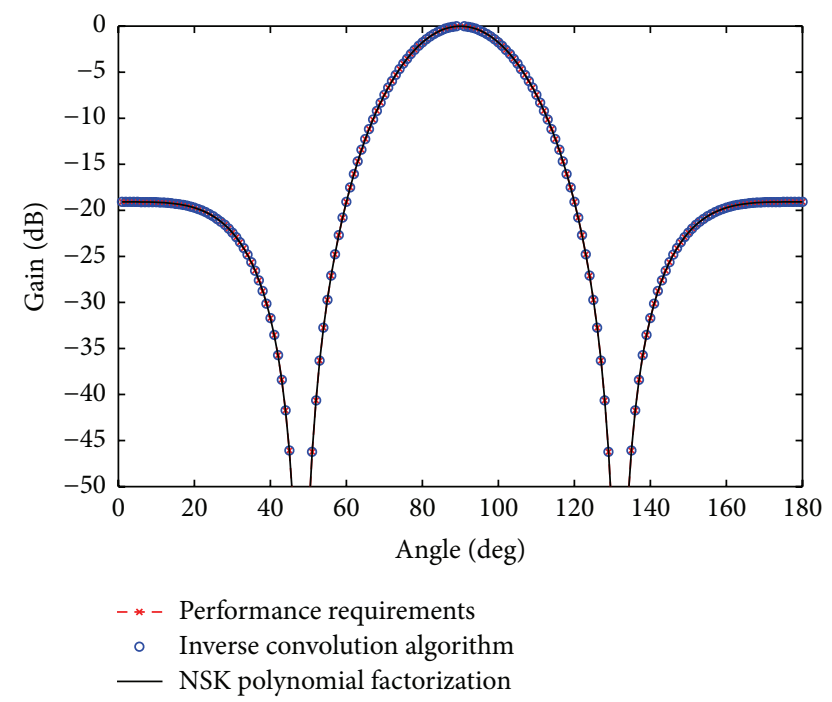

Figure 4: Performance of different algorithms.

The polynomial factorization result obtained by the proposed algorithm is $P_{V}(x)=\left(1+x+x^{2}\right)\left(1+x+x^{2}\right)$; that is, $P_{T}(x)=1+x+x^{2}, P_{T}(x)=1+x+x^{2}$.

The results obtained by the inverse convolution algorithm in [22] are the same.

Figure 3 shows the transmitting and receiving array design. It can be noticed that 3 antennas located at positions 0,1 , and 2 are required and the system degrees of freedom are 5. Figure 4 gives the comparison of the beam patterns. The MIMO radar antenna array design algorithm using NSK polynomial factorization has the same performance as the inverse convolution algorithm, satisfying the requirements of performance. The main lobe width is 25.95 degrees and the first side lobe is $-19.08 \mathrm{~dB}$.

In conclusion, when the system virtual equivalent array is nonuniform, the proposed algorithm is applicable and the performance is the same as the algorithm in [9].
Example 2 (uniform equivalent array design of bistatic MIMO radar). Consider $\mathbf{G}_{V}=(1,1, \ldots, 1)$ and $P_{V}(x)=\sum_{i=0}^{14} x^{i}$.

In [22], the monostatic MIMO radar cannot be obtained by inverse convolution algorithm; the solution by an unconstraint least square approximation is $\mathbf{G}_{T}=\mathbf{G}_{R}=(0.7493$, $0.4084,0.3367,0.312,0.3127,0.3366,0.4083,0.7494)$.

The polynomial factorization result obtained by the proposed algorithm is $P_{V}(x)=\left(1+x+x^{2}\right)\left(1+x^{3}+x^{6}+x^{9}+x^{12}\right)$ :

$$
\begin{aligned}
& \text { Design 1: } P_{T}(x)=1+x+x^{2}, P_{R}(x)=1+x^{3}+x^{6}+ \\
& x^{9}+x^{12} . \\
& \text { Design 2: } P_{T}(x)=1+x^{3}+x^{6}+x^{9}+x^{12}, P_{R}(x)= \\
& 1+x+x^{2} .
\end{aligned}
$$

The results obtained by the inverse convolution algorithm in [9] are the same.

Figure 5(a) shows that the solution by inverse convolution algorithm is $\mathbf{G}_{V}=(0.5615,0.6120,0.6714,0.7426$, $0.8368,0.9699,1.1947,1.8783,1.1949,0.9699,0.8363,0.7435$, $0.6712,0.6120,0.5616)$, which does not satisfy the requirements. Figures 5(b) and 5(c) show the equivalent array vector of the two designs by the proposed algorithm which is $\mathbf{G}_{V}=(1,1,1,1,1,1,1,1,1,1,1,1,1,1,1)$, which satisfies the requirements. In design 1 , the transmitting antennas are located at positions 1,2 , and 3 and the receiving antennas are located at positions $0,3,6$, and 9 . In design 2 , the transmitting antennas are located at positions $0,3,6$, and 9 and the receiving antennas are located at positions 0,1 , and 2 . Though the beam patterns of the two designs are the same, they are also seen as two different designs for they have different transmitting and receiving gains. Figure 6 shows the beam pattern of the different designs. The main lobe width of the approximate solution is 7.97 degrees and the first side lobe is $-15.64 \mathrm{~dB}$. The main lobe width of the proposed algorithm is 6.79 degrees and the first side lobe is $-13.13 \mathrm{~dB}$, which satisfies the requirements of system performance.

In conclusion, when the system equivalent array is uniform but the exact results for monostatic MIMO radars cannot be obtained by inverse convolution algorithm in [9], the proposed algorithm can be applied to get the ideal results and the performance is the same as the algorithm's in [17].

Example 3 (nonuniform system equivalent array design of bistatic MIMO radar). Consider $\mathbf{G}_{T}=(1,2,3,3,2,1)$ and $P_{V}(x)=1+2 x+3 x^{2}+3 x^{4}+2 x^{5}+x^{6}$.

The equivalent array is nonuniform, so the algorithm in [17] cannot be applied. Additionally, the inverse convolution cannot get the accurate solutions. By the proposed algorithm, we can get the factorization: $P_{V}(x)=(1+x)\left(1+x^{2}\right)\left(1+x+x^{2}\right)$ :

$$
\begin{aligned}
& \text { Design 1: } P_{T}(x)=1+x, P_{R}(x)=\left(1+x^{2}\right)\left(1+x+x^{2}\right) . \\
& \text { Design 2: } P_{T}(x)=1+x^{2}, P_{R}(x)=(1+x)\left(1+x+x^{2}\right) . \\
& \text { Design 3: } P_{T}(x)=1+x+x^{2}, P_{R}(x)=(1+x)\left(1+x^{2}\right) .
\end{aligned}
$$

(The swapping of the transmitter and receiver will not be described here.)

Figure 7 shows three possible transmitting and receiving arrays, whose equivalent array vector is $\mathbf{G}_{V}=(1,2,3,3,2,1)$. It can be noticed that 8 antennas are required for design 1 and 

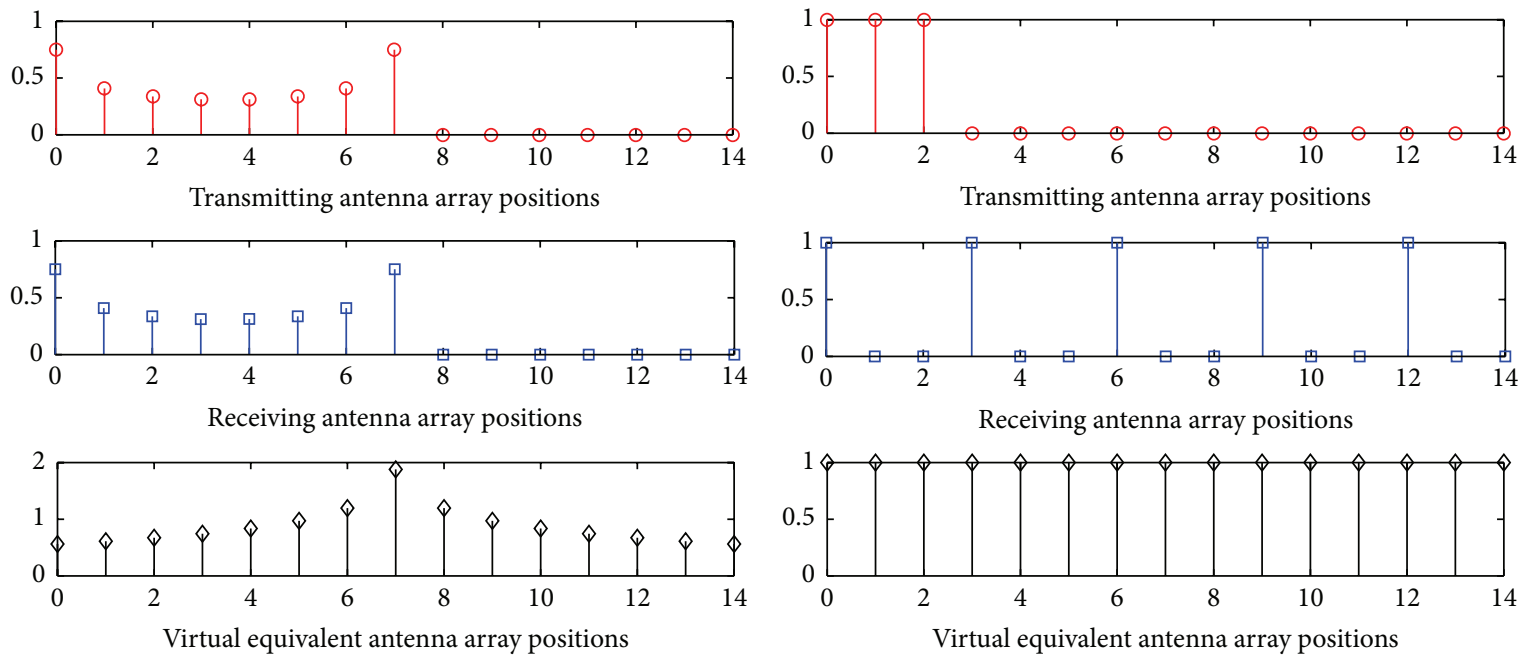

(a) Design of inverse convolution

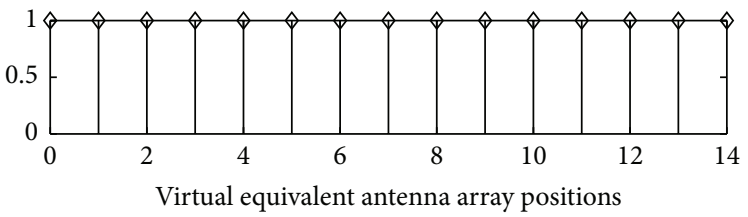

(b) Design 1 of NSK polynomial factorization
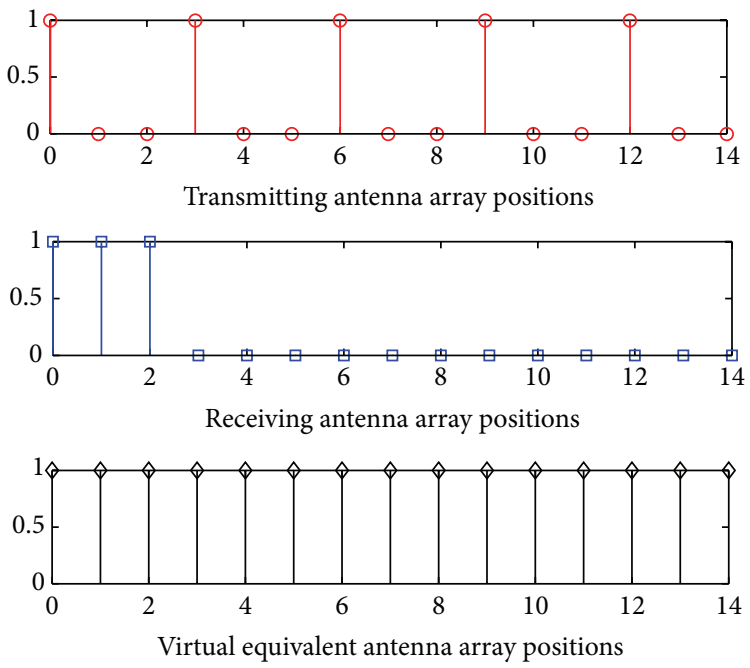

(c) Design 2 of NSK polynomial factorization

FIGURE 5: Design of inverse convolution and NSK polynomial factorization.

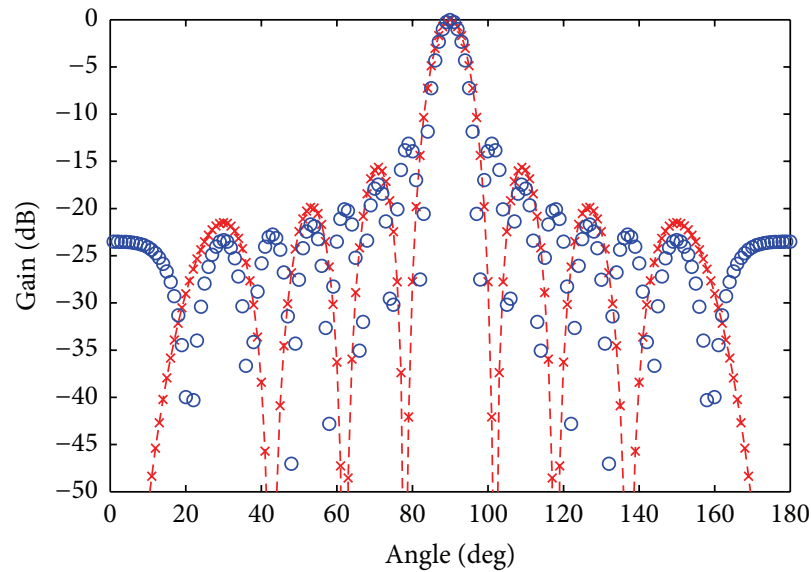

-* - Performance requirements

- Inverse convolution algorithm

(a) Comparison between inverse convolution and performance requirements

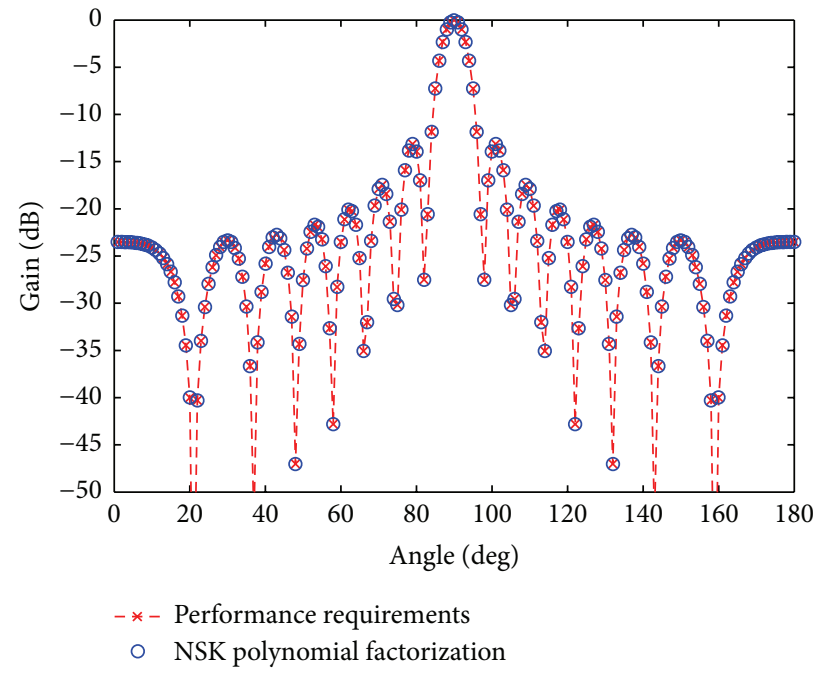

(b) Comparison between NSK polynomial factorization and performance requirements

Figure 6: Performance of different algorithms. 

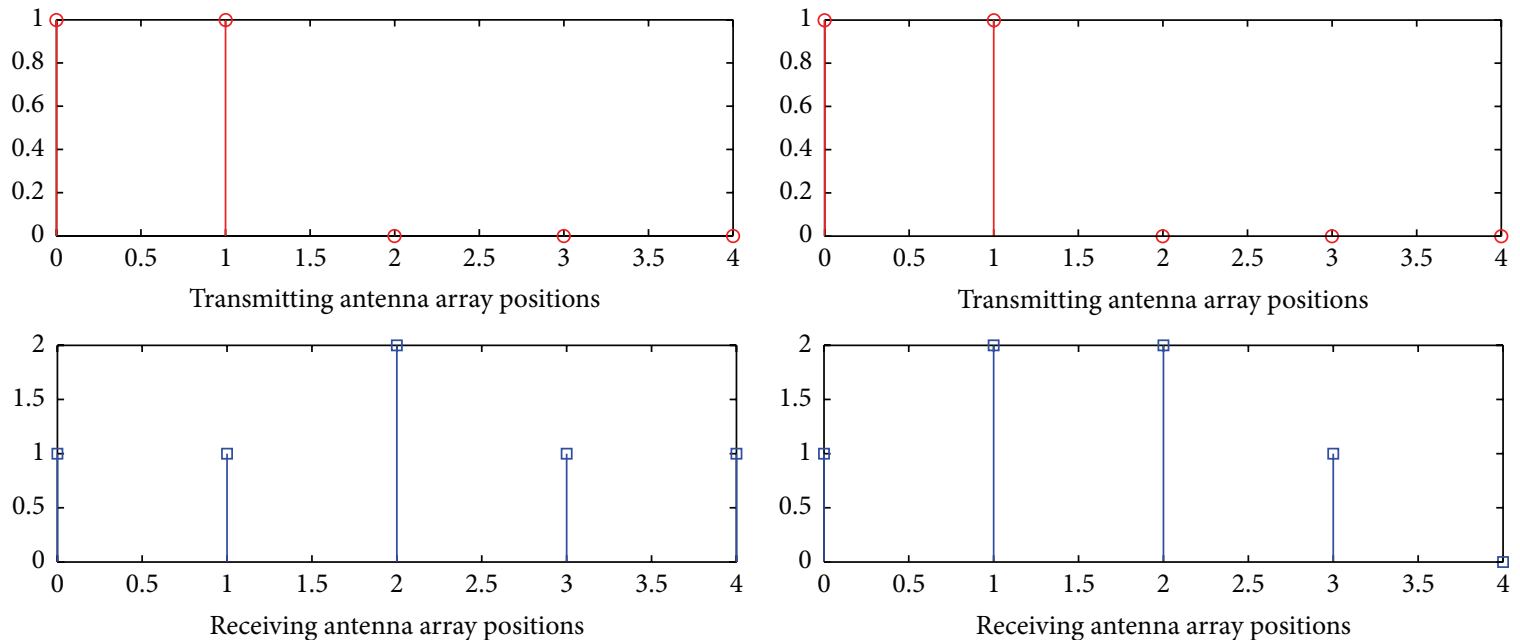

(a) Design 1

(b) Design 2
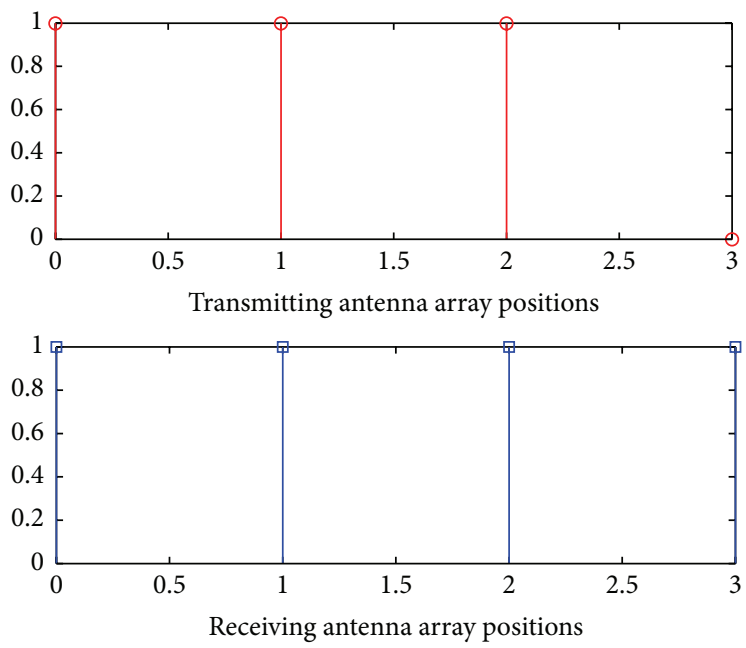

(c) Design 3

FIGURE 7: Designs of NSK polynomial factorization.

design 2 and 7 antennas for design 3. All the designs have 7 degrees of freedom. Figure 8 gives the comparison of beam patterns, showing the arrays designed by NSK polynomial factorization satisfy the requirements.

In conclusion, when the system equivalent array is nonuniform and the exact results cannot be obtained by inverse convolution algorithm in [9], the proposed algorithm can be applied to get the ideal results which satisfy the requirements of system performance.

\section{Conclusion}

In order to extend the application scope of the existing algorithms for MIMO radar array design, an algorithm using NSK polynomial factorization is proposed in the paper. The algorithm describes the MIMO radar polynomial model in detail and lists the steps of the proposed algorithm. For a given system performance, the algorithm can be accomplished to design all the transmitting and receiving arrays which can

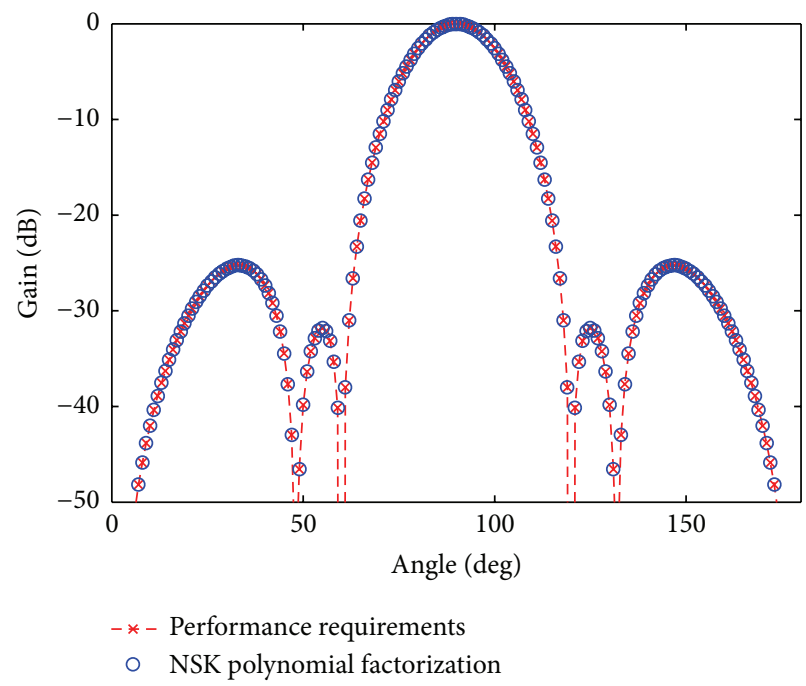

FIGURE 8: Comparison between NSK polynomial factorization and performance requirements. 
meet the requirements. Simulation examples under different situations are given to verify the wide use of the algorithm and indicate that the proposed algorithm can be widely used no matter whether the transmitting and receiving arrays are bistatic or monostatic and the system equivalent antenna arrays are nonuniform or uniform.

In fact, some multichannel radar system antenna array design is similar to the MIMO radar system as long as the emission signal does not produce space power synthesis, such as the use of different frequency transmit signal. The algorithm proposed by the paper can be also used [9].

We are aware that the synthesized pattern with angle scanning capability must be taken into account in actual MIMO antenna arrays. This topic will be further investigated in our subsequent work.

\section{Conflict of Interests}

The authors declare that there is no conflict of interests regarding the publication of this paper.

\section{Acknowledgments}

This work was supported by the National Science and Technology Major Project under Grant no. 2011ZX03003-003-02 and 863 Program under Grant no. 2012AA01A502 and no. 2012AA01A505.

\section{References}

[1] E. Fishler, A. Haimovich, R. Blum, D. Chizhik, L. Cimini, and R. Valenzuela, "MIMO radar: an idea whose time has come," in Proceedings of the IEEE Radar Conference, pp. 71-78, Philadelphia, Pa, USA, April 2004.

[2] A. Hassanien and S. A. Vorobyov, "Transmit/receive beamforming for MIMO radar with colocated antennas," in Proceedings of the IEEE International Conference on Acoustics, Speech, and Signal Processing (ICASSP '09), pp. 2089-2092, IEEE, Taipei, Taiwan, April 2009.

[3] C. Yang, Y. Yao, J. Yu, and X. Chen, "Novel compact multiband MIMO antenna for mobile terminal," International Journal of Antennas and Propagation, vol. 2012, Article ID 691681, 9 pages, 2012.

[4] J. Zheng, Y. Li, and Z. Feng, "Impact of mutual coupling and polarization of antennas on BER performances of spatial multiplexing MIMO systems," International Journal of Antennas and Propagation, vol. 2012, Article ID 795205, 12 pages, 2012.

[5] K. W. Forsythe, D. W. Bliss, and G. S. Fawcett, "Multiple-input multiple-output (MIMO) radar: performance issues," in Proceedings of the 38th Asilomar Conference on Signals, Systems and Computers, vol. 1, pp. 310-315, IEEE, Pacific Grove, Calif, USA, November 2004.

[6] F. Belfiori, W. Van Rossum, and P. Hoogeboom, "Coherent MIMO array design with periodical physical element structures," IEEE Antennas \& Wireless Propagation Letters, vol. 10, pp. 1341-1344, 2011.

[7] A. A. Gorji, R. Tharmarasa, and T. Kirubarajan, "Optimal antenna allocation in MIMO radars with collocated antennas," IEEE Transactions on Aerospace \& Electronic Systems, vol. 50, no. 1, pp. 552-558, 2014.
[8] A. A. Gorji, T. Kirubarajan, and R. Tharmarasa, "Antenna allocation for MIMO radars with collocated antennas," in Proceedings of the 15th International Conference on Information Fusion (FUSION '12), pp. 424-431, IEEE, Singapore, July 2012.

[9] Y. Su, Y. T. Zhu, and W. X. Yu, "Multi-channel radar array design method and algorithm," Science China Information Sciences, vol. 53, no. 7, pp. 1470-1480, 2010.

[10] B. Sun, H. Chen, D. Yang, and X. Li, "Antenna selection and placement analysis of MIMO radar networks for target localization," International Journal of Distributed Sensor Networks, vol. 2014, Article ID 769404, 10 pages, 2014.

[11] J. Li and P. Stoica, MIMO Radar Signal Processing, Wiley, IEEE, 2009.

[12] C. Duofang, C. Baixiao, and Q. Guodong, "Angle estimation using ESPRIT in MIMO radar," Electronics Letters, vol. 44, no. 12, pp. 770-771, 2008.

[13] C.-Y. Chen and P. P. Vaidyanathan, "Minimum redundancy MIMO radars," in Proceedings of the IEEE International Symposium on Circuits and Systems (ISCAS '08), pp. 45-48, IEEE, Seattle, Wash, USA, May 2008.

[14] A. Kirschner, U. Siart, J. Guetlein, and J. Detlefsen, "A designalgorithm for MIMO radar antenna setups with minimum redundancy," in Proceedings of the IEEE International Conference on Microwaves, Communications, Antennas and Electronic Systems (COMCAS '13), vol. 143, pp. 1-5, Tel Aviv, Israel, October 2013.

[15] F. Belfiori, W. Van Rossum, and P. Hoogeboom, "Coherent MIMO array design with periodical physical element structures," IEEE Antennas and Wireless Propagation Letters, vol. 10, pp. 1341-1344, 2011.

[16] J. Dong, R. Shi, Y. Guo, and W. Lei, "Antenna array design in MIMO radar using cyclic difference sets and genetic algorithm," in Proceedings of the 10th International Symposium on Antennas, Propagation \& EM Theory (ISAPE '12), pp. 26-29, IEEE, Xian, China, October 2012.

[17] W.-Q. Wang, H. Shao, and J. Cai, "MIMO antenna array design with polynomial factorization," International Journal of Antennas and Propagation, vol. 2013, Article ID 358413, 9 pages, 2013.

[18] P. F. Sammartino, D. Tarchi, and C. J. Baker, "MIMO radar topology: a systematic approach to the placement of the antennas," in Proceedings of the International Conference on Electromagnetics in Advanced Applications (ICEAA '11), pp. 114-117, IEEE, September 2011.

[19] S. K. Mitra, K. Mondal, M. K. Tchobanou, and G. J. Dolecek, "General polynomial factorization-based design of sparse periodic linear arrays," IEEE Transactions on Ultrasonics, Ferroelectrics, \& Frequency Control, vol. 57, no. 9, pp. 1952-1966, 2010.

[20] S. K. Mitra, M. K. Tchobanou, and M. I. Bryukhanov, "A general method for designing sparse antenna arrays," in Proceedings of the European Conference on Circuit Theory and Design, vol. 2, pp. II/263-II/266, IEEE, September 2005.

[21] R. S. Elliott, Antenna Theory and Design, Prentice-Hall, Englewood Cliffs, NJ, USA, 1981.

[22] T. T. Taylor, "Design of line-source antennas for narrow beamwidth and low side lobes," Transactions of the IRE Professional Group on Antennas and Propagation, vol. 3, no. 1, pp. 1628, 1955.

[23] C.-Y. Tseng and L. J. Griffiths, "A simple algorithm to achieve desired patterns for arbitrary arrays," IEEE Transactions on Signal Processing, vol. 40, no. 11, pp. 2737-2746, 1992. 
[24] M. Skolnik, Introduction to Radar Systems, McGraw-Hill, New York, NY, USA, 1980.

[25] E. W. Cheney, Introduction to Approximation Theory, Chelsea, London, UK, 1982.

[26] R. R. Coifman, R. Rochberg, and G. Weiss, "Factorization theorems for Hardy spaces in several variables," Annals of Mathematics, vol. 103, no. 3, pp. 611-635, 1976. 


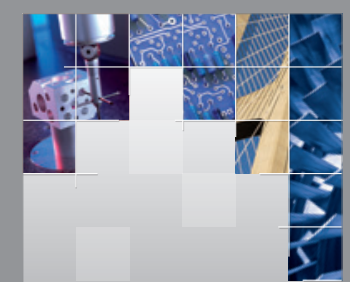

\section{Enfincering}
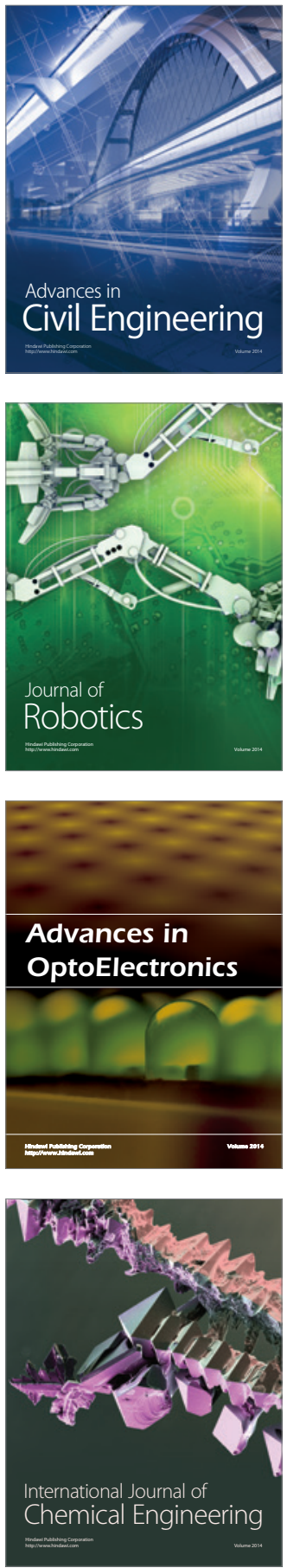

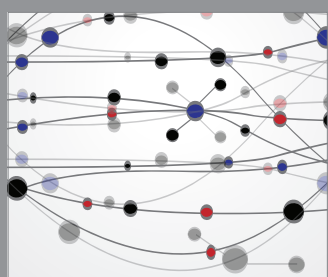

The Scientific World Journal

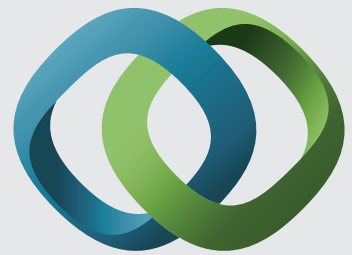

\section{Hindawi}

Submit your manuscripts at

http://www.hindawi.com
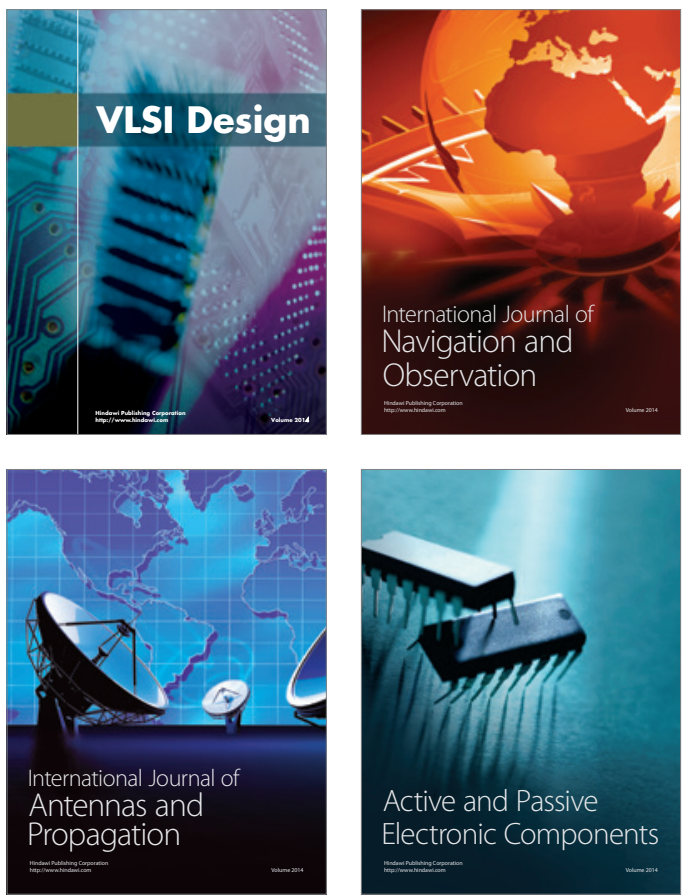
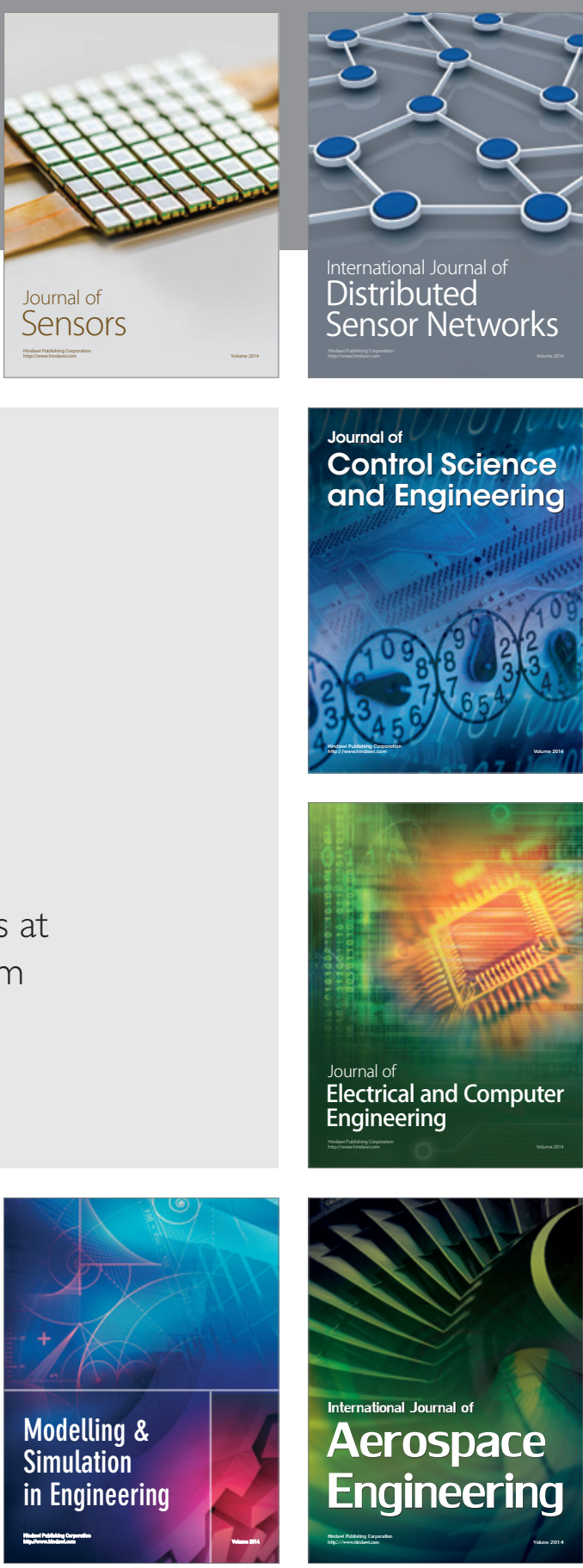

International Journal of

Distributed

Sensor Networks

Journal of

Control Science

and Engineering
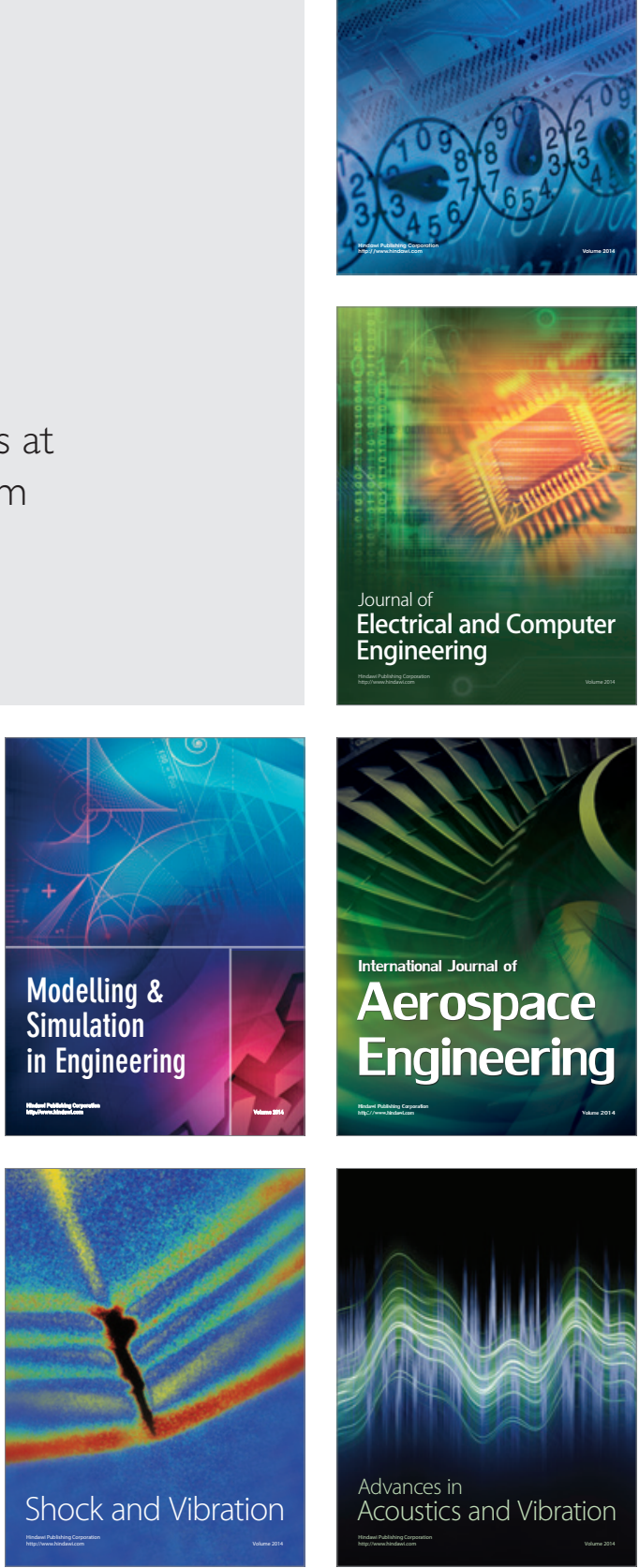